\title{
Performance of Multihop Relaying System in Cognitive Radio Networks
}

\author{
Nada Ebaid \\ Faculty of Electronic \\ Engineering \\ El-Menofiya University, Egypt.
}

\author{
Mona shokair \\ Faculty of Electronic \\ Engineering \\ El-Menofiya University, Egypt.
}

\author{
Sami A. El Dolil \\ Faculty of Electronic \\ Engineering \\ El-Menofiya University, Egypt.
}

\begin{abstract}
This paper introduces multihop relay system where the benefits of spatial diversity are achieved from the concurrent reception of signals that have been transmitted by multiple terminals. Amplify and forward cooperative relays will be used in this system. It will be obvious that multihop system can effectively mitigate the performance deterioration caused by fading, without imposing delay or bandwidth expansion. The channel models are compared through the simulations to prove that multihop system is better than single hop system where it gives low symbol error rate and therefore the performance will be improved. Maximum Ratio Combining will be used. Analysis of different hops system will be made. Different modulations will be applied. From simulation results it will be obvious that symbol error rate will be reduced when using multihop than single hop.
\end{abstract}

\section{Keywords}

Cognitive radio, cooperative relay, multihop, modulation, amplify and forward relay.

\section{INTRODUCTION}

Cognitive radio network is proposed to solve limiting of the spectrum problems. Cognitive radio, is proposed by Mitola [1] in which it enables the XG network to use spectrum in dynamic manner to be capable of sensing and adapting to environments. In this network, unlicensed user referred to as secondary user(SU) can access the unused spectrum of the licensed user referred to as primary user(PU).In practical, secondary users want to send its data on the licensed frequency of the primary users but secondary users must not compromise the communication of the primary user. If the spectrum is available secondary user sent its data and if not secondary user wait for random time and make sensing again and so on until it can access the spectrum. There are different methods of sensing that secondary user can use which are explained in [2]. Cooperation transmission improves spatial diversity for the same frequency band as the original idea of it comes from the basic relay model that consists of three terminals: source $S$, relay $R$, and destination $D$ [3]. By enabling a set of cooperating relays to forward received information, this regime exploits spatial diversity through cooperation among distributed antennas belonging to multiple terminals in wireless networks. The referenced cooperative protocols involve only a single intermediate relay terminal between the source and destination terminals. These protocols can be easily extended to the case of multiple relaying terminals in parallel or multihop scenarios where each destination is instead a relay itself, and is paired with a different cooperating terminal with which it cooperates to transmit to the actual destination or next relay. However, there is no obvious extension to multihop cooperation scenarios where the signal from the source is directly received and used by many relays further along the transmission path, or to asymmetric multihop cooperation scenarios where a relay further along the transmission path does not use the source to relay information to its destination. This paper introduces the concept of multihop diversity; where the benefits of spatial diversity are achieved from the concurrent reception of signal that has been transmitted by multiple previous terminals. Spatial diversity techniques that can effectively mitigate the performance deterioration caused by fading, without imposing delay or bandwidth expansion, are clearly desirable from efficiency and system usability points of view. Spatial diversity is obtained when signals transmitted from antennas separated far enough to experience independent fading channels. Since it is not always practical to deploy multiple antennas at a mobile due to size or other constraints, user cooperation has been proposed [4].This scheme exploits the broadcast nature of wireless networks where the communications channel is shared between multiple terminals [5].In cognitive relaying, after detecting a "spectral hole", the source broadcasts the signal to the destination and the relays then the relays forward the signal to the destination [6]. These protocols determine what the individual relay should do, decode-and-forward (DF), amplify-and-forward (AF), or compress-and-forward (CF), after receiving the signal [7].

In this paper, the performance of cognitive multihop relay network will investigated under different number of hops that use repetition-based transmission with $\mathrm{M}$ relays. One hop, two hops and three hops will be introduced. Different types of modulation will be applied.

The rest of this paper will be organized as follows: Section 2 presented one hop system model. Section 3 introduced two hops system model. Three hops system model will be investigated in Section 4 Multihop system model will be studied in Section 5 Simulation results will be made in Section 6 .Finally, conclusions will be made.

\section{One Hop System Model}

The essence of cooperative communication (CC) is to exploit (1) the wireless broadcast advantage and (2) the relaying capability of other nodes so as to achieve higher throughput, lower transmission error, or other objectives in transmission(8). A secondary network will be introduced with a source node (S) transmits data to a destination node(D) through the help of direct link and relay link (R) as illustrated in Figure 1. 


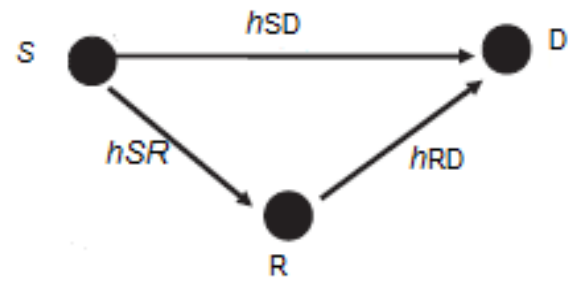

Fig.1. One hop System model.

Transmission data in secondary system using cooperative relay in CRN consists of two phases. In the first phase, the source broadcast messages to the destination and the relay. In the second phase, the relay amplifies source messages and forwards to the destinations. Amplify and forward protocol is used. Finally, the destination combines the signals from the source and the relay with maximum ratio combine (MRC) to decode source messages. There can be two received signal models. The first is without Interference from the Primary User and the other is with Interference from the Primary User. Here without Interference from the Primary User operation mode will be studied [6].It will be considered that secondary user correctly detect no primary user activity. Firstly in general case secondary user source will send its signal to the destination and to the relay. The signal received at destination and relay are given by, $y_{S D}=\sqrt{P_{1}} h_{S D} x+n_{S D}$

$y_{S R}=\sqrt{P_{1}} h_{S R} x+n_{S R}$

Where $x$ is the zero-mean and unit-energy transmitted symbol, $P_{1}$ is the source energy, $h_{S D}$ is the channel response between the source and the destination, $h_{S R}$ is the channel response between the source and the relay and $n_{S D} \sim C N\left(0, \sigma_{n_{i t}}^{2}\right)$ and $n_{i t} \sim C N\left(0, \sigma_{\mathrm{n}_{i \mathrm{t}}}^{2}\right)$ are the complex Gaussian noise samples. The relay, after normalization, forwards the received signal to the destination. Therefore the received signal at the destination from the relay is donated by,

$y_{\mathrm{RD}}=\sqrt{P_{2}} h_{R D} x_{i}+n_{R D}$

Where $h_{R D}$ is the known channel response between the receiver and the relay, $P_{2}$ is the relay energy and $n_{R D} \sim C N$ $\left(0, \sigma_{\mathrm{RD}}^{2}\right)$ is the complex Gaussian noise [6]. Substituting $x_{i}$ in (3) give

$y_{R D}=\sqrt{\frac{p_{1} p_{2}}{p_{1}\left|h_{R D}\right|^{2}+\sigma^{2} S R}} h_{R D} h_{S R} S+n_{R D}^{\tilde{R}}$

Where $n_{r i}^{\sim} \sim C N\left(0, \sigma_{n R D}^{2}\right)$ and $\sigma_{n R D}^{2 \sim}=\frac{p_{2}\left|h_{R D}\right|^{2} \sigma_{n_{S R}}^{2}}{p_{1}\left|h_{R D}\right|^{2}+\sigma_{n_{R D}}^{2}}+\sigma_{n_{R D}}^{2}$

\section{Two Hops System Model}

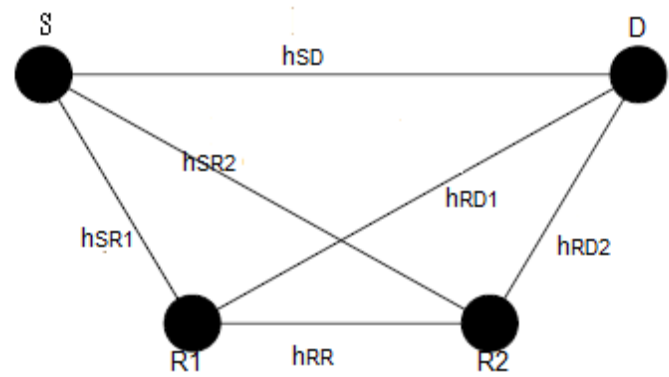

Fig.2. Two hops system model.
Figure 2 shows two hops system model. In this system, two relays will be used. Space diversity will be achieved through sending the source signal to the destination with the help of four paths. Maximal ratio combining (MRC) will be used at the destination. The transmission operation in the two hops system will take three time slots. In the first time slot, the source will transmit its signal to the destination (D), relay one (R1) and relay two (R2). The received signals at the destination relay one and relay two are given by,

$y_{S D}=\sqrt{P_{1}} h_{S D} \quad X+n_{S D}$

$y_{S R 1}=\sqrt{P_{1}} h_{S R 1} X+n_{S R 1}$

$y_{S R 2}=\sqrt{P_{1}} h_{S R 2} X+n_{S R 2}$

As $P_{1}$ is the source energy, $h_{S D}$ is the channel response between the source and the destination, $h_{S R 1}$ is the channel response between the source and the $\mathrm{R} 1, h_{S R 2}$ is the channel response between the source and $\mathrm{R} 2$ and $n_{S D}$ and $n_{i t}$ are the complex Gaussian noise samples. In the second time slot R1 and R2 amplify the source signal with suitable gain and then forward it to the destination. In the same time slot $\mathrm{R} 2$ sent the amplified signal to R2. The amplification gain of R1 and R2 in the second time slot are

$\beta_{1}=\sqrt{\frac{\mathrm{P}_{2}}{\mathrm{P}_{1}\left|h_{S R 1}\right|^{2}+n_{S R 1}}}$

$\beta_{2}=\sqrt{\frac{\mathrm{P}_{3}}{\mathrm{P}_{1}\left|h_{S R 2}\right|^{2}+n_{S R 2}}}$

The received signals at destination from $\mathrm{R} 1$ and $\mathrm{R} 2$ are

$y_{\mathrm{RD} 1}=\beta_{1} h_{R D 1} y_{S R 1}+n_{R D 1}$

$y_{\mathrm{RD} 2}=\beta_{2} h_{R D 2} y_{S R 2}+n_{R D 2}$

The received signal at $\mathrm{R} 2$ from $\mathrm{R} 1$ is given by,

$y_{\mathrm{RR}}=\beta_{1} h_{R R} y_{S R 1}+n_{R R 12}$

In the third time slot R2 will amplify the received signal from $\mathrm{R} 1$ with amplification gain $\beta_{22}$.

$\beta_{22}=\sqrt{\frac{\mathrm{P}_{3}}{\mathrm{P}_{2}\left|h_{R R}\right|^{2}+n_{R R 12}}}$

The received signal at destination from $\mathrm{R} 2$ is

$y_{\mathrm{RD} 22}=\beta_{22} h_{R D 2} \quad y_{\mathrm{RR}}+n_{R D 2}$

At the destination all the received signals during the three time slots will be companied using MRC.

\section{Three Hops System Model}

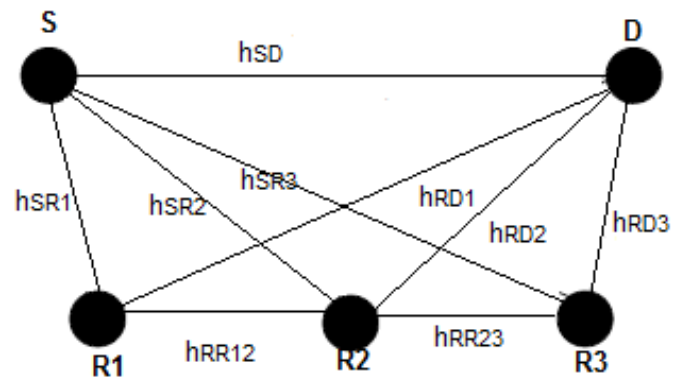

Fig.3. Three hops system model.

By increasing the number of relays from two to three relays the number of paths between source and destination will increase to seven paths and so space diversity will be achieved. Figure 3 shows three hops system model. The 
transmission operation here will take four time slots and also MRC will be used at the destination.

At the first time slot, $\mathrm{S}$ will transmit its signal to destination, $\mathrm{R} 1, \mathrm{R} 2$ and $\mathrm{R} 3$.the received signals at them are

$$
\begin{aligned}
& y_{S D}=\sqrt{P_{1}} h_{S D} \quad X+n_{S D} \\
& y_{S R 1}=\sqrt{P_{1}} h_{S R 1} X+n_{S R 1} \\
& y_{S R 2}=\sqrt{P_{1}} h_{S R 2} \quad X+n_{S R 2} \\
& y_{S R 3}=\sqrt{P_{1}} h_{S R 3} \quad X+n_{S R 3}
\end{aligned}
$$

At the second time slot, the relays will amplify the received signals at $1^{\text {st }}$ time slot with amplification gains $\beta_{1}, \beta_{2}$ and $\beta_{3}$

$\beta_{1}=\sqrt{\frac{\mathrm{P}_{2}}{\mathrm{P}_{1}\left|h_{S R 1}\right|^{2}+n_{S R 1}}}$

$\beta_{2}=\sqrt{\frac{\mathrm{P}_{3}}{\mathrm{P}_{1}\left|h_{S R 2}\right|^{2}+n_{S R 2}}}$

$\beta_{3}=\sqrt{\frac{\mathrm{P}_{4}}{\mathrm{P}_{1}\left|h_{S R 3}\right|^{2}+n_{S R 3}}}$

Where $\mathrm{P}_{1}, \mathrm{P}_{2}, \mathrm{P}_{3}$ and $\mathrm{P}_{4}$ are the power of $\mathrm{S}, \mathrm{R} 1, \mathrm{R} 2$ and $\mathrm{R} 3$. Relays then forward the amplified signals to the destination and also each relay transmit its amplified signal to the next relay to it. The received signals at the destination from R1, $\mathrm{R} 2$ and $\mathrm{R} 3$ are

$$
\begin{aligned}
& y_{\mathrm{RD} 1}=\beta_{1} h_{R D 1} y_{S R 1}+n_{R D 1} \\
& y_{\mathrm{RD} 2}=\beta_{2} h_{R D 2} y_{S R 2}+n_{R D 2} \\
& y_{\mathrm{RD} 3}=\beta_{3} h_{R D 2} y_{S R 3}+n_{R D 3}
\end{aligned}
$$

The received signals at $\mathrm{R} 2$ from $\mathrm{R} 1$ and at $\mathrm{R} 3$ from $\mathrm{R} 2$ are

$y_{\mathrm{RR} 12}=\beta_{1} h_{R R 12} y_{S R 1}+n_{R R 12}$

$y_{\mathrm{RR} 23}=\beta_{2} h_{R R 23} y_{S R 2}+n_{R R 23}$

At the third time slot each relay amplify the received signal from the previous relay with amplification gains $\beta_{22}$ and $\beta_{33}$

$\beta_{22}=\sqrt{\frac{\mathrm{P}_{3}}{\mathrm{P}_{2}\left|h_{R R 12}\right|^{2}+n_{R R 12}}}$

$\beta_{33}=\sqrt{\frac{\mathrm{P}_{4}}{\mathrm{P}_{3}\left|h_{R R 23}\right|^{2}+n_{R R 23}}}$

And then forward it to the destination and to the next relay. The received signals at the destination from $\mathrm{R} 2$ and $\mathrm{R} 3$ are

$y_{\mathrm{RD} 22}=\beta_{22} h_{R D 2} y_{\mathrm{RR} 12}+n_{R D 2}$

$y_{\mathrm{RD} 33}=\beta_{33} h_{R D 3} y_{\mathrm{RR} 23}+n_{R D 3}$

The received signal from R2 to R3 after amplification operation with $\beta_{22}$ is

$y_{\mathrm{RR} 233}=\beta_{22} h_{R R 23} y_{\mathrm{RR} 12}+n_{R R 23}$

At the fourth time slot the received signal $y_{\mathrm{RR} 23}$ at R3 will be amplified with $\beta_{33}$ and then forward it to the destination. Then the received signal at destination is

$y_{\mathrm{RD} 333}=\beta_{33} h_{R D 3} y_{\mathrm{RR} 233}+n_{R D 3}$

Then the seven signals from different seven paths will be companied to give better result than two hops than one relay system and this will be introduced in the simulation results.

\section{Multihop System Model}

A multi-hop wireless network where there are multiple concurrent uncast sessions will be considered. The transmitted signal from the source to the destination may traverse multiple hops in the network. It will be considered that the transmitters are able to transmit concurrently without any interference [8].There is two types of special network introduced for one source-destination pair and $M$ relays. In the first type the source node transmit its signal to destination and to $\mathrm{M}$ relay nodes. Then $\mathrm{M}$ relay nodes amplify the signal and forward it to the destination. Without relay nodes share an edge with the edge between themselves .For the second type of network the source node transmit its signal to destination and to $\mathrm{M}$ relay nodes. Similarly, $M$ relay nodes amplify the signal and forward it to the destination but relay nodes share an edge with the edge between themselves as each node transmit its signal to the next node in the network [9]. Consider a secondary network in which a source node transmits data to a destination node through the help of an amplify-and-forward, multi-branch, multi-hop relay system with $\mathrm{M}$ relays, in which the relays broadcast to other relays as well as the destination with access to the licensed bands only available in the absence of primary activity as shown in figure 4.The amplification gains used at the relays will be adapted with the received signal that will be amplified. To obtain this gain the power of the received signal will be computed and then with the help of the relay power an average power will be introduced. This average power will be used at amplification operation [9]. The source and the relays transmit on orthogonal channels; therefore, do not cause interference with each other. A time orthogonal system is assumed. The receiving terminals use maximum ratio combining (MRC) to combine all of the signals that they have previously received. All channels are Rayleigh fading channels with additive white Gaussian noise (AWGN).

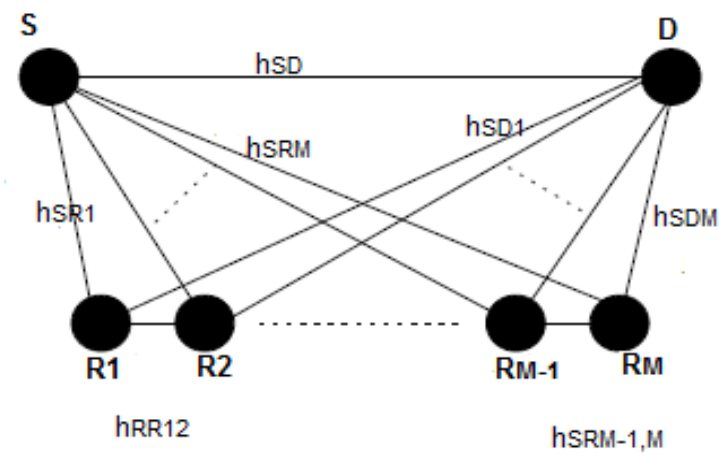

Fig.4. Multihop system model

The channel coefficient for the channel from transmitting terminal $i$ to receiving terminal $j$ is denoted by $h_{i, j}$ where $i=s$ for the source; $i=1,2$. .For relay $i ; j=1,2, \mathrm{M}$ for relay $j$; and $j=d$ for the destination. Channel coefficient distribution is denoted by $h_{i, j} \sim\left(0, \sigma_{i, j}^{2}\right)$ since $h_{i, j}$ is complex Gaussian with variance $\sigma_{i, j}^{2}$, it follows that $\left|h_{i, j}\right|^{2}$ is an exponential RV with a mean of $\sigma_{i, j}^{2}$. The channels are considered to be slow fading, so that the coefficients are constant over the time duration of one symbol, that is, over one time slot. The AWGN component at terminal $j$, added to the signal transmitted from terminal $i$, is denoted by $n_{i, j}$.It is also a zero-mean complex Gaussian $\mathrm{RV}$, its variance per dimension is $N_{i, j} / 2$, and its distribution is denoted by $\mathcal{C N}\left(0, \mathrm{~N}_{\mathrm{i}, \mathrm{j}}\right)$. The channel coefficients and AWGN signals are all mutually independent. The 
transmission operation here will take $\mathrm{M}+1$ time slots to sent source signal with the help of $\mathrm{M}$ relays. In time slot 0 , the source broadcasts the signal with the help of its average power $P_{1}$. The transmitted signal from the source is equal to $\sqrt{P_{1}} x$ where $x$ is the set of possible signals for the source to transmit. The relays $(j=1,2, \ldots)$ and destination $(j=d)$ receive the signals $y_{s, j}=\sqrt{P_{1}} h_{s, j} x+n_{s, j}$ for $j=1,2, \ldots, \mathrm{M}$, $d$. In time slot 1 , relay1 broadcasts $x_{1}=\beta_{1} y_{s, 1}$ to the next relays and to the destination where $\square_{l}$ is chosen so that the average power used by relay 1 is equal to $\square_{l}$, a predetermined desired value of power [10]. In the same time slot 1 relay 2 will broadcast its signal to the next relays and to the destination. This operation is the same at other relays from relay 1 to relay $M$ but relay $M$ will only transmit its signal to the destination as illustrated in fig. 4.The amplifying gain $\square_{l}$, is given by $\square_{I}=\sqrt{\frac{\square_{2}}{\left.\square_{l} h_{\square \square l}\right|^{2}+\square_{\square, l}}}$ and now general equation for this multi hop system at any time slot will be introduced. At $\square \square h$ relay the amplifying gain $\beta_{\mathrm{j}}$ is $\beta_{\mathrm{j}}=\sqrt{\frac{\mathrm{P}_{\mathrm{j}}}{\left.\square_{\square} \mathrm{h}_{\mathrm{i}, \mathrm{j}}\right|^{2}+\mathrm{n}_{\mathrm{i}, \mathrm{j}}}}$ and then the broadcast signal from $j$ th relay terminals after amplification is $x_{j}=\beta_{j} y_{j-1, j}$. The received signals at terminals $\mathrm{M}=\mathrm{j}+1 \ldots \mathrm{M}+1$ are

$\mathrm{y}_{\mathrm{j}, \mathrm{M}}=\mathrm{h}_{\mathrm{j}, \mathrm{M}} \mathrm{x}_{\mathrm{j}}+\mathrm{n}_{\mathrm{j}, \mathrm{M}}$.

\section{Simulations Results}

In this section, the numerical result will be presented to study the symbol error rate versus signal to noise ratio for multi-hop system with $\mathrm{M}$ relays. Amplify and forward relays will be used. A maximal ratio combiner is assumed at receiving terminals for multi-hop channels with diversity. The total frame duration equal to $200 \mathrm{~ms}$. The number of packet equal to 5000.The SER versus SNR will be presented under different kinds of modulation in the one hop system and at multihop system. Modulation schemes that studied are binary phase shift keying (BPSK), Quadrature phase shift keying (QPSK) and 8-phase shift keying (8PSK) as shown in Figure 5 .

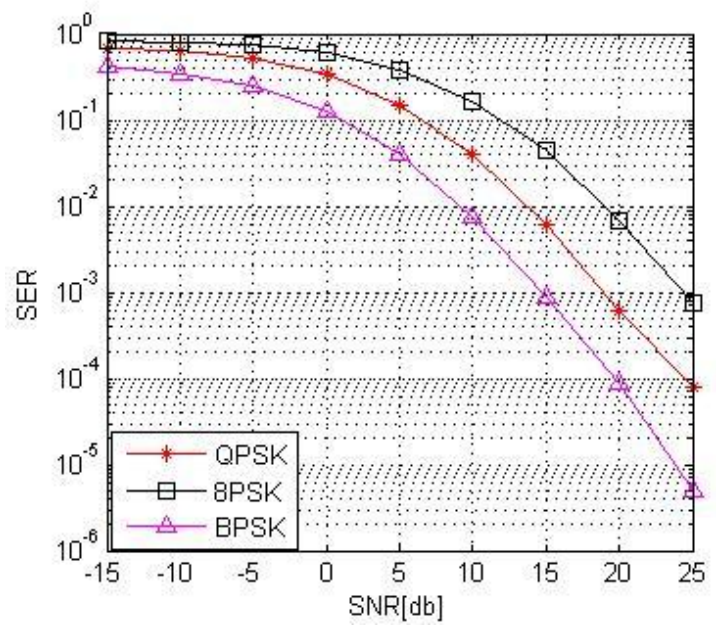

Fig.5. SER versus SNR using different kinds of modulation.

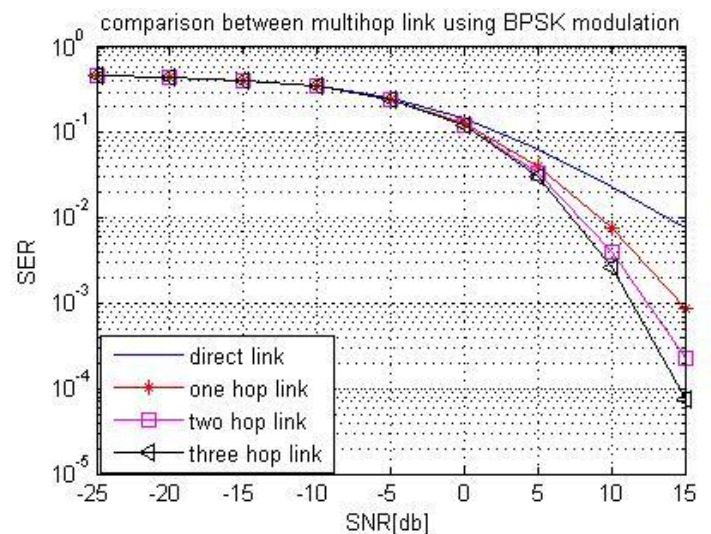

Fig.6. SER for the multihop system using BPSK modulation.

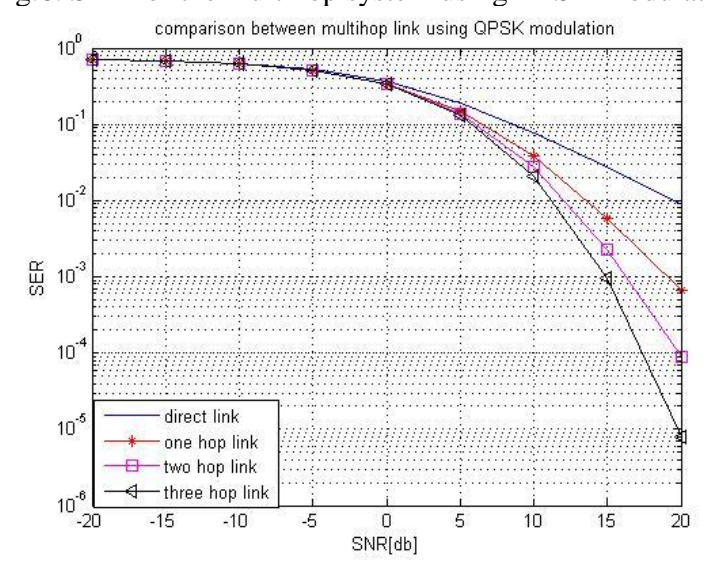

Fig.7. SER for the multihop system using QPSK modulation.

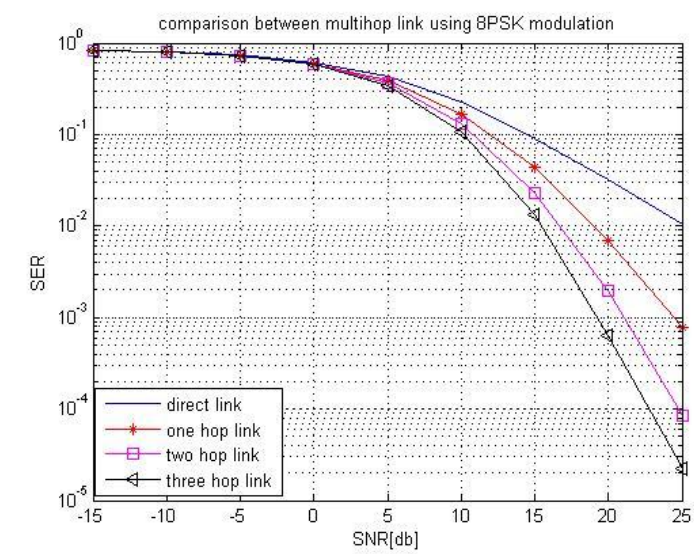

Fig.8. SER for the multihop system using 8PSK modulation.

Figures 6, 7, and 8 show the relations between SER vs. SNR (dB) when BPSK, QPSK and 8PSK, respectively. It will be cleared from these figures that multiple hops gives better performance than single hop at any different modulation schemes.

\section{Conclusions}

Multihop wireless communication system with amplify and forward cooperative relays was introduced. At various modulation schemes, the performance of multihop relaying system was investigated. It can be obvious that using multihop technique improve the performance of the system and as we increase the number of relays between source and destination, the symbol error rate decreases but if this number increase over specified number, the interference will increase. To avoid this interference, the system will be more complex. 


\section{References}

[1] J. Mitola and Q. Maguire, "Cognitive Radio: Making Software Radios More Personal ", IEEE Communications Society, PP.13-18, vol. 6, Aug. 1999.

[2] H. Tran, T. Duong and H.Zepernick, "Performance Analysis of Cognitive Relay Networks Under Power Constraint of Multiple Primary Users", Global Telecommunications Conference, IEEE, pp. 1-6, Dec. 2011.

[3] Q.Zhang, J.Jia and J.Zhang, "Cooperative Relay to Improve Diversity in Cognitive Radio Networks", IEEE Communications Magazine, vol. 47, pp.111 -117, Feb. 2009.

[4] M. Yu and Jing Li, " Is Amplify-and-Forward Practically Better than Decode-and-Forward or Vice Versa?", IEEE International Conference ,vol. 3,pp. 365 - 368,2005.

[5] J.Boyer, D.Falconer and H.Yanikomeroglu, "Multihop Diversity in Wireless Relaying Channels", IEEE transaction on wireless communication, vol. 52, no. 10, Oct. 2004.
[6] A.Zafar, M.Alouini, Y.Chen and R.Radaydeh, "Minimizing Symbol Error Rate for Cognitive Relaying with Opportunistic Access", 7th International ICST Conference on Cognitive Radio Oriented Wireless Networks and Communications, pp. 153 - 157, June. 2012.

[7] J.Jiang, J.Thompson, P.Grant and N.Goertz, "Practical Compress-And-Forward Cooperation for The Classical Relay Network", 17th European Signal Processing Conference Scotland,Aug. 2009.

[8] S.Sharma, Y. Thomas Hou,Hanif D. Sherali and SastryKompella, "Cooperative Communications in Multi-hop Wireless Networks: Joint Flow Routing and Relay Node Assignment", IEEE Journal, vol. 30 ,pp. 254 $-262,2012$.

[9] S. Agnihotri, S. Jaggi, and M. Chen, "Amplify-andForward in Wireless Relay Networks", IEEE Transactions, vol. 53, pp. $3302-3318,2007$.

[10] C. Conne, MinChulJu, Zhihang Yi, H.Song, and Il-Min Kim, "SER Analysis and PDF Derivation for Multi-Hop Amplify-and-Forward Relay Systems", IEEE Transaction on Wireless Communication, vol. 58, no. 8, Aug. 2010. 